\section{Neurodermitisdiagnose per Pricktest?}

Milde Formen der atopischen Dermatitis lassen sich nicht immer leicht von anderen Hauterkrankungen abgrenzen. Auf der Suche nach einer möglichen diagnostischen Hilfe haben Deutsche Allergologen nun die Juckbohne untersucht.

B islang gibt es keine etablierten Biomarker, mit denen sich eine atopische Dermatitis (AD) zweifelsfrei diagnostizieren lässt. In der Vergangenheit wurde unter anderem das diagnostische Potenzial von Trichomen der Juckbohne (Mucuna pruriens) diskutiert, die in der Lage sind, einen histaminunabhängigen Pruritus zu erzeugen. Im Vergleich zu gesunden Kontrollen nahm die Intensität des Juckreizes bei AD-Patienten langsamer ab. Ärzte von der Berliner Charité haben nun untersucht, wie sich Juckreiz, Quaddeln und Hauterscheinung bei AD-Patienten und gesunden Probanden unterscheiden, wenn diese entweder mit Juckbohnen oder mit einem klassischen Histamin-Pricktest provoziert werden.

Die Erytheme, die bei einem Histamin-Pricktest nach 20 Minuten gemes- sen wurden, waren bei AD-Patienten signifikant kleiner als bei Gesunden (18 \pm $2 \mathrm{~mm}$ vs. $32 \pm 3 \mathrm{~mm}$; p < 0,01). Zudem hält der Juckreiz bei AD-Patienten nach Juckbohnen-Provokation länger an (14 von 22 Patienten $>30$ Minuten) als bei Hautgesunden (3 von $18>30$ Minuten). Bei der maximalen Juckreiz-Intensität unterschieden sich beide Gruppen nicht, wenngleich die Neurodermitiker mehrere Juckreiz-Qualitäten nannten (u.a. pulsierend, in Wellen, zwickend, kalt). Diese beiden Parameter konnten eine $\mathrm{AD}$ unabhängig voneinander vorhersagen: der Erythemdurchmesser mit einer Sensitivität von $59 \%$ und einer Spezifität von $78 \%$, die Juckreizdauer entsprechend mit $64 \%$ und $83 \%$. Statistische Auswertungen ergaben, dass beide Parameter kombiniert sogar noch besser abschnitten. So ergab sich zum Beispiel

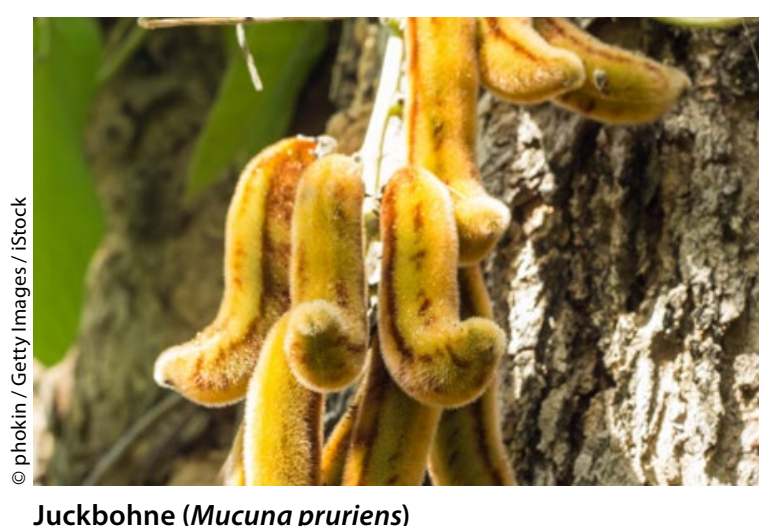

eine Spezifität von $94 \%$, wenn beide Kriterien gleichzeitig vorlagen.

Fazit: Die klassische Positivkontrolle beim Pricktest mit Histamin könnte tatsächlich noch mehr diagnostische Informationen zu Tage fördern als bislang bekannt. Ein Juckbohnen-induzierter Pruritus, der über 30 Minuten anhält, und histamininduzierte Erytheme auf läsionsfreier Haut mit einem Durchmesser unter $20 \mathrm{~mm}$ nach 20 Minuten deuten auf eine $\mathrm{AD}$ hin. Sebastian Lux

Hawro T et al. Skin provocation tests may help to diagnose atopic dermatitis. Allergy 2016; 71 : $1745-52$

\title{
SLIT: gute Lebensqualität und hohe Adhärenz
}

Die Adhärenz zu einer Behandlung wird umso besser sein, je mehr ein Patient einen Therapieeffekt etwa in Form einer verbesserten Lebensqualität spürt. Eine Praxisstudie liefert Daten zu diesem postulierten Zusammenhang.

W ie bei jeder Langzeittherapie ist die Therapieadhärenz auch unter der meist über drei Jahre angelegten sublingualen Immuntherapie (SLIT) in den meisten Fällen nicht optimal. Als eine wichtige Ursache wird ein möglicherweise vom Patienten nicht wahrgenommener Therapieeffekt diskutiert. Eine monozentrische und retrospektiv durchgeführte Praxisstudie überprüfte deshalb einen möglichen Zusammenhang zwischen Lebensqualität und Adhärenz unter einer SLIT an einem pädiatrischen Patientenkollektiv. Dazu wurde den Eltern der von 2009 bis 2014 behandelten Kinder ein Fragebogen zugeschickt, der die Lebensqualität mithilfe des generischen SF-12 Health Surveys anonym er- fasste. Anonymisierte Praxisunterlagen lieferten Daten zur Therapieadhärenz. Von den 393 verschickten SF-12-Fragebögen kamen 201 ausgefüllt zurück. In der gewichteten und zusammenfassenden Auswertung ergab sich bei den körperlichen Domänen ein Score von 49,3 (plusminus 7,0) und bei den mental-psychischen Domänen ein Score von 52,6 (plusminus 7,2) - Werte, die alters- und geschlechtsadaptiert vergleichbar auch in bevölkerungsbasierten Befragungen in Deutschland gefunden werden. $73 \mathrm{der}$ 104 Patienten (70,2\%), die bis zum Jahr 2011 eine SLIT begonnen hatten, blieben über drei Therapiejahre adhärent.

Dass die Kinder in dieser Studie eine Lebensqualität wie der Bevölkerungs- durchschnitt haben, spricht letztlich für einen spürbaren Behandlungseffekt der SLIT, schreiben die Autoren in der Diskussion. Denn bekanntermaßen ist die Lebensqualität von Allergikern schlechter als die der Allgemeinbevölkerung, nachgewiesen auch mithilfe des SF12-Fragebogens. Die in dieser Querschnittsstudie gefundene Adhärenz von $70 \%$ liegt über dem in anderen Studien ermittelten Durchschnittswert. Das könnte ein Indiz dafür sein, dass die Patienten einen Behandlungseffekt gespürt haben, so die Autoren.

Fazit: Die Therapietreue zu einer SLIT steigt, wenn die Patienten eine verbesserte Lebensqualität wahrnehmen. Das legen die retrospektiven Daten aus einer pädiatrisch ausgerichteten Praxis nahe.

Dr. Barbara Kreutzkamp

Lemberg ML et al. Importance of quality of life for adherence to sublingual immunotherapy. BioMed Res Int 2016; 2016: 5186765 